\title{
AFuzzy Unifying Format Basedon Triangular Fuzzy Numbers and Intuitionistic Fuzzy Numbers
}

\author{
Zamali Tarmudi ${ }^{1}$, Norfazillah Matmali ${ }^{2}$, Nowyannie Willie D. Tamsin ${ }^{3} \&$ \\ MohdLazim Abdullah ${ }^{4}$ \\ 1, 2 \& 3 Faculty of Computer and Mathematical Sciences, UniversitiTeknologi MARA (UiTM) Sabah Branch, \\ Locked Bag 71, 88997 Kota Kinabalu, Sabah, Malaysia.) \\ ${ }^{4}$ (School of Informatics and Applied Mathematics, Universiti Malaysia Terengganu (UMT), \\ 21030 Kuala Terengganu, Terengganu, Malaysia.)
}

\begin{abstract}
The aim of this study is to propose the use of the fuzzy systematic technique as aunifying format for analyzing diverse input data sources using both triangular fuzzy numbers (TFNs) and intuitionistic fuzzy numbers (IFNs). It focuses on converting three different data sources into the unifying format which consists of crisp values, interval values as well as linguistic expressions. This study utilizedthe experience of experts in order to construct the membership functions for the crisp datasets, while the advantages of both the TFNs and IFNs based onthe cost-benefitcriterion were employed respectively for datasets whichoccurred naturally in interval and linguistic forms. An empirical analysis related to the multi-criteria decision-making (MCDM) problem was employ to demonstrate the feasibility and suitability of the proposed approach. The results show that the approach is well-suited, capable and efficient as a unifying tool for both TFNs and IFNs, particularly for solving MCDM problems. It offers a versatile judgment, has clear procedures and also has great potential as a unifying tool for three input data sources which arediverse in nature. Thus, it helps decision-makers (DMs) make their decisionswith ease and in a systematic manner.
\end{abstract}

Keywords: Fuzzy unifying format, input data sources, Intuitionistic Fuzzy Numbers (IFNs), multi-criteria decision-making (MCDM), Triangular Fuzzy Numbers (TFNs)

\section{Introduction}

Recently, the unifying format has been viewed with increasing concern by researchers in the decisionmaking process. The requirements are vital and more significant in real-world applications because most of the input datasets are uncertain, vague and imprecise in nature. Although many researchers are aware and concernedabout these issues, effort has not really been concentrated on direct discussions regarding how evaluators can unify input datasets from different sources.

Multi-criteria decision-making (MCDM) in particular, has also played an increasingly vital role in the unifying format particularly in real-world applications such as in biomass studies, medical image analysis, computing, industrial engineering and business management [1-5]. In a fuzzy MCDM problem for example, the decision-makers (DMs) evaluate the importance of the criteria and sub-criteria using direct assignmentof linguistic variables. According to [6], in order to deal with the qualitative and quantitative original datasets, two methods were utilized where: i) qualitative data in the form of fuzzy linguistic variables isused to construct the linguistic values and derive its corresponding value to the triangular fuzzy numbers, and ii) quantitative data, through consultations with DMs, is used to estimate reasonable data input based on their expertise and experiences. Currently, the evolution of the MCDM probleminvolves diverse input datasets. It may include different data sources such as crisp values, interval values as well as linguistic expression or forms.

However, classical MCDM methods are unrealistic because they only consider crisp datasets. For example, in a job selection environment which has three alternatives - clerk, teacher and businessman - each alternative would involve four criteria: salary, security, location and benefits. In this context, crisp datasets cannot be evaluated by DMs for the location criteria due to the influence ofa variety of other factors (i.e., subcriteria). This will cause uncertainty in input datasets such as unquantifiable, unobtainable and incomplete information, and partial ignorance [7]. Moreover, in this classical method,diversity of input datasets (e.g., different in nature) is also less discussed in the decision-making process.

In MCDM problems which utilize the cost-benefitcriterionsuch as supplier selection [8] and project evaluation [9], there are various problems involving diverse variables which may emerge. Both the qualitative and quantitative cost-benefit-based techniquesare applicable and promising as compared to the classical method. Since this classical method cannot overcome this aspect of uncertainty, a prospective method has been introduced [10]. Therefore, fuzzy methods, probabilistic information and DM's attitudes under uncertainty has been proposed by [11]. For example, [12] introduced the preferences element under uncertainty for DMs to 
make a decision in a simple manner. The MCDM model can be built up based on evidence sourcesto be combined, discounting operations and reliability of the evaluation method [13].In this paper, we present the systematic approach using thefuzzy unifying formatfor diverse input datasets for MCDM using both TFNs and IFNs. Based on past works, many studies have adopted the fuzzy unifying format to overcome certain problems. The fuzzy unifying format has been adopted in managing feasibility and performance in Indoor Environment Quality (IEQ). Butfuzzy unifyingneeds a higher computational of the fuzzy formalism. Hence, the unifying controller has benefited in accommodateand overcome the traditional controller [14]. Besides, [15] have introduced fuzzy unification to overcome in agent communication languagesdue to the missing parameters and mismatchof predicates and parameters.

The main objective of this paper is to propose the use of the fuzzy systematic technique as a unifying format for analyzing diverse MCDM input data sources using both triangular fuzzy numbers (TFNs) and intuitionistic fuzzy numbers (IFNs). The structure of this paper is as follows; Firstly, we provide a brief reviewof basic important definitions and properties of fuzzy unifying(Section 2). Then, in Section 3, we explain the proposed unifying procedures for fuzzy and intuitionistic fuzzy approachesby using triangular fuzzy numbers (TFNs) and intuitionistic fuzzy numbers (IFNs). To make our proposed method clearer, an empirical example is presented in Section 4for both the fuzzy and intuitionistic fuzzy analyses based on the same case study. Finally, Section 5 containsbrief conclusions.

\section{Preliminaries}

In this section, some basic important definitions and properties regarding fuzzy sets, intuitionistic fuzzy numbers, triangular fuzzy numbers, and the cost-benefit criterion for TFNs and IFNs arebriefly reviewed. These basic definitions and notations below will be used throughout this paper.

Definition 1. A fuzzy set $A$ in the universe of discourse $X=\left\{x_{1}, x_{2}, \ldots, x_{n}\right\}$ is defined as [16]:

$$
A=\left\{\left\langle x, \mu_{\tilde{A}}(x)\right\rangle x \in X\right\}
$$

which is characterized by the membership function $\mu_{\tilde{A}}(x): X \rightarrow[0,1]$, where $\mu_{\tilde{A}}(x)$ indicates the membership degree of the element $X$ to the set $A$.

Definition 2.A triangular fuzzy number $\tilde{t}$ is defined by a triplet $(a, b, c)$. The membership function is defined as [16]:

$$
\mu_{\tilde{t}}(x)= \begin{cases}\frac{x-a}{b-c}, & a \leq x \leq b \\ \frac{c-x}{c-b}, & b \leq x \leq c \\ 0, & \text { otherwise }\end{cases}
$$

The triangular fuzzy number is based on a triplet form; the minimum possible value $a$, the most possible value $b$ and the maximum possible value $c$. Table 1 shows the example of linguistic variables for ratings and their TFNs.

Table 1.The Linguistic Variable for Ratings and their TFNs

\begin{tabular}{lc}
\hline \multicolumn{1}{c}{ Linguistic terms } & TFNs \\
\hline Very low $(V L)$ & $(0,0.1,0.2)$ \\
Low $(L)$ & $(0.1,0.2,0.3)$ \\
Medium low $(M L)$ & $(0.2,0.3,0.4)$ \\
Medium $(M)$ & $(0.3,0.5,0.7)$ \\
Medium high $(M H)$ & $(0.6,0.7,0.8)$ \\
High $(H)$ & $(0.7,0.8,0.9)$ \\
Very high $(V H)$ & $(0.8,0.9,1.0)$ \\
\hline
\end{tabular}

To deal with the diversity of input data sources in MCDM problems, theviews of expertsare utilized in order to construct the membership functions for the crisp data, while the advantages of TFNs basedon the costbenefit criterion were employed for the data both naturally in interval and in linguistic form. Thus, some definitions and related properties based on the cost-benefitcriterion are as follows:

Definition 3. Let $\tilde{u}_{i j}=\left(a_{i j}, b_{i j}, c_{i j}\right)=(i=1,2, \ldots, n ; j=1,2, \ldots, m)$, thus we employedthe method by [17]and is given as:

$$
\widetilde{U}=\left(\tilde{u}_{i j}\right)_{m \times n}
$$




$$
\begin{gathered}
\text { where } \tilde{u}_{i j}=\left(\frac{a_{i j}}{M}, \frac{b_{i j}}{M}, \frac{c_{i j}}{M}\right) ; i=1,2, \ldots, n ; j \in \omega_{1} \\
\tilde{u}_{i j}=\left(\frac{N-c_{i j}}{N}, \frac{N-b_{i j}}{N}, \frac{N-a_{i j}}{N}\right) ; i=1,2, \ldots, n ; j \in \omega_{2} \\
M=\max _{i} c_{i j}, j \in \omega_{1}(\text { benefit - criteria }) ; N=\max _{i} c_{i j}, j \in \omega_{2}(\text { cost }- \text { criteria })
\end{gathered}
$$

The higher the value of $\tilde{u}_{i j}$ for $\omega_{1}$, the better it is for DMs and the lower the value of $\tilde{u}_{i j}$ for $\omega_{2}$, the better it is for the DMs. This unifying process preserves the property in the range of $[0,1]$.

Meanwhile for IFNs based onthecost-benefit criterion, the definition and properties are as below:

Definition 4. Let $X$ be an ordinary finite non-empty set. An IFS (Intuitionistic fuzzy set)in A is an expression A given by [18]:

$$
A=\left\{\left\langle x, \mu_{x}(x), v_{x}(x)\right\rangle x \in X\right\}
$$

where $\mu_{x}(x): X \rightarrow[0,1] ; v_{x}(x): X \rightarrow[0,1]$ with the condition: $0 \leq \mu_{x}(x)+v_{x}(x) \leq 1$ for all $x$ in $X$. The numbers $\mu_{x}(x)$ and $v_{x}(x)$ denote, respectively, the degree of the membership and the degree of the nonmembership of the element $x$ in the set $A$. The notation of IFS ' $A$ ' is defined as follows:

$$
\pi_{x}(x)=1-\mu_{x}(x)-v_{x}(x) ; \pi_{x}: X \rightarrow[0,1]
$$

$\pi_{x}(x)$ represents the degree of hesitation or intuitionistic index or non-determinacy of $x$ to $A$. Therefore, for ordinary fuzzy sets, the degree of hesitation $\pi_{x}(x)=0$.

For convenience of computation, an intuitionistic fuzzy number (IFN) is viewed as

$$
\alpha=\left(\mu_{\alpha}, v_{\alpha}, \pi_{\alpha}\right) \text { where } \mu_{\alpha} \in[0,1], v_{\alpha} \in[0,1] ; \mu_{\alpha}+v_{\alpha} \leq 1, \pi_{\alpha}=1-\mu_{\alpha}-v_{\alpha}
$$

Table 2 shows the example of linguistic terms for ratings and their IFNs.

Table 2.The Linguistic Variable for Ratings and theirIFNs

\begin{tabular}{llll}
\hline Linguistic terms & \multicolumn{3}{c}{ IFNs } \\
\hline Very low $(V L)$ & $(0.98$, & 0.02, & $0.00)$ \\
Low $(L)$ & $(0.75$, & 0.15, & $0.10)$ \\
Medium low $(M L)$ & $(0.65$, & 0.25, & $0.10)$ \\
Medium $(M)$ & $(0.50$, & 0.35, & $0.15)$ \\
Medium high $(M H)$ & $(0.35$, & 0.55, & $0.10)$ \\
High $(H)$ & $(0.15$, & 0.75, & $0.10)$ \\
Very high $(V H)$ & $(0.02$, & 0.98, & $0.00)$ \\
\hline
\end{tabular}

In MCDM problems, the values of different criteria have different dimensions. The crisp numbers in the unifying decision need to be standardized in order to eliminate interference in the final results. Generally, there are two types of criteria, the benefit as well as the cost. The higher the value of the benefit type, the better it is, while in the cost type, it is the opposite.

Definition 5. Conversion between exact values to IFNs by [19].Let $\dot{a}_{i j}$ be the exact value, for the benefit type, and the standardizing formulaeare listed as follows:

$$
b_{i j}=\frac{\dot{a}_{i j}}{\sqrt{\sum_{i=l}^{m}\left(\dot{a}_{i j}\right)^{2}}} ; i=1,2, \ldots, m ; j \in \omega_{1} \text { (benefit-criterion) }
$$

For the cost type, the standardizing formulaeare listed as follows:

$$
b_{i j}=\frac{\left(1 / \dot{a}_{i j}\right)}{\sqrt{\sum_{i=l}^{m}\left(1 / \dot{a}_{i j}\right)^{2}}} ; i=1,2, \ldots, m ; j \in \omega_{2} \text { (cost-criterion) }
$$

Standardized precise numbers can be transformed into IFNs $a_{i j}=\left(\mu_{i j}, v_{i j}, \pi_{i j}\right)$. Consider the following: 


$$
\mu_{i j}=b_{i j}, \quad v_{i j}=1-b_{i j}, \quad \pi_{i j}=0 .
$$

Definition 6.Conversion between interval values to IFNsby [19].Let $a_{i j}$ be the interval value, for the benefit type, and the standardizing formulaeare listed as follows:

$$
\begin{gathered}
b_{i j}^{L}=\frac{a_{i j}^{L}}{\sqrt{\sum_{i=l}^{m}\left(a_{i j}^{U}\right)^{2}}} \text { and } b_{i j}^{U}=\frac{a_{i j}^{U}}{\sqrt{\sum_{i=l}^{m}\left(a_{i j}^{L}\right)^{2}}} ; \\
i=1,2, \ldots, m ; j \in \omega_{1} \text { (benefit-criterion) }
\end{gathered}
$$

For the cost type, the standardizing formulae are listed as follows:

$$
\begin{gathered}
b_{i j}^{L}=\frac{1 / a_{i j}^{U}}{\sqrt{\sum_{i=l}^{m}\left(1 / a_{i j}^{L}\right)^{2}}} \text { and } b_{i j}^{U}=\frac{1 / a_{i j}^{L}}{\sqrt{\sum_{i=l}^{m}\left(1 / a_{i j}^{U}\right)^{2}}} ; \\
i=1,2, \ldots, m ; j \in \omega_{2} \text { (cost-criterion) }
\end{gathered}
$$

Standardized precise numbers can be transformed into IFNs $a_{i j}=\left(\mu_{i j}, v_{i j}, \pi_{i j}\right)$. Consider the following:

$$
\mu_{i j}=\mathrm{b}_{i j}^{L}, \quad v_{i j}=1-\mathrm{b}_{i j}^{U}, \quad \pi_{i j}=\mathrm{b}_{i j}^{U}-\mathrm{b}_{i j}^{L} .
$$

Definition 7. Cost-benefit criterion for IFNs by[20].We consider an intuitionistic fuzzy number (IFN), and this is viewed as $\alpha=\left(\mu_{\alpha}, v_{\alpha}, \pi_{\alpha}\right)$ where $\mu_{\alpha} \in[0,1], v_{\alpha} \in[0,1] ; \mu_{\alpha}+v_{\alpha} \leq 1, \pi_{\alpha}=1-\mu_{\alpha}-v_{\alpha}$. Then IFNs for the cost criterionare

$$
\bar{\alpha}=\left(v_{\alpha}, \mu_{\alpha}, \pi_{\alpha}\right)
$$

where $\mu_{\alpha} \in[0,1], v_{\alpha} \in[0,1] ; v_{\alpha}+\mu_{\alpha} \leq 1, \pi_{\alpha}=1-v_{\alpha}-\mu_{\alpha}$, and IFNs for the benefitcriterionare

$$
\alpha=\left(\mu_{\alpha}, v_{\alpha}, \pi_{\alpha}\right)
$$

where $\mu_{\alpha} \in[0,1], v_{\alpha} \in[0,1] ; \mu_{\alpha}+v_{\alpha} \leq 1, \pi_{\alpha}=1-\mu_{\alpha}-v_{\alpha}$

\section{Our Proposed Unifying Process using Both the TFNs and IFNs}

For easy understanding of our proposed unifying approach in MCDM problems, we perform the unifying process using 5 main procedures as below.

\subsection{Problem definitions and formulation}

Let $U$ be the decision matrix (where the entry $u_{i j}$ represents the rating of alternative $\mathrm{A}_{\mathrm{i}}$ with respect to criterion $C_{j}$ ), and $\mathrm{W}$ as the weight vector (where $w_{j}$ represents the weight of criterion $C_{j}$ ) can be concisely expressed as: $U=\left[u_{i j}\right]$ and $W=\left(w_{j}\right)$; where $i=1, \ldots, m$ and $j=1, \ldots, n$. Where $A_{i}(i=1, \ldots, m)$ is $m$ alternatives and $\mathrm{n}$ criteria $C j(j=1, \ldots, n)$. Please take note that this step - problem definitions and formulation is needed for both the TFN and IFN applications.

\subsection{Unifying Process}

Two methods were employed to unify the non-homogenous input data sources to derive the score values.

i. The original data is represented by a crisp value, as per feedback from the expert; the membership function was constructed to derive the membership values for TFNs, while equations (7) - (12) are utilized for IFNs.

ii. The original data is represented by interval and linguisticterms, and we employed both (3) - (4) for TFNs, while (13) -(14) have been utilized for IFNs. Both TFNs and IFNs are based on cost-criterion or benefitcriterion,respectively.

\subsection{Performance Matrix}

The performance matrix of the problem is calculated according to the nature of input datasets given as $\tilde{U}$ for

TFNs and $\tilde{\tilde{U}}$ for IFNsas shown below 


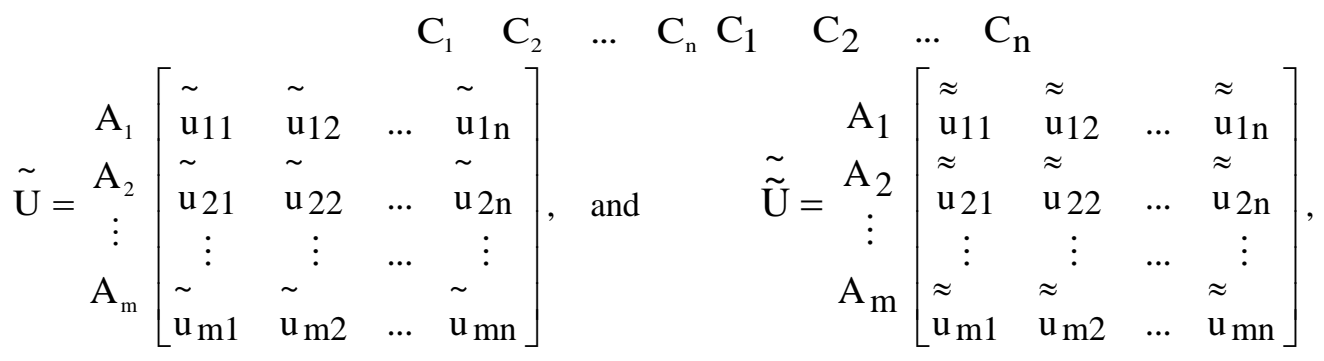

where

$A_{1}, A_{2}, \ldots, A_{m}$ are possible alternatives,

$C_{1}, C_{2}, \ldots, C_{n}$ are criteria measured using TFNs and IFNs, respectively

$\tilde{u}_{i j}=\left(a_{i j}, b_{i j}, c_{i j}\right)$ isthe triplet of the TFNs and $\underset{\mathrm{u}}{\approx \mathrm{ij}}=\left(\mu_{i j}, v_{i j}, \pi_{i j}\right)$ is IFNs

\subsection{Performance index}

To evaluate the performance index (PI), the vertex method by [21] is used with reference to ideal solutions [22]. Let $\tilde{x}=\left(a_{x}, b_{x}, c_{x}\right)$ and $\tilde{y}=\left(a_{y}, b_{y}, c_{y}\right)$ be two positive TFNs, then the vertex method defines the distance between them as:

$$
d(\tilde{x}, \tilde{y})=\sqrt{\frac{1}{3}\left[\left(a_{x}-a_{y}\right)^{2}+\left(b_{x}-b_{y}\right)^{2}+\left(c_{x}-c_{y}\right)^{2}\right]}
$$

We define the positive ideal solution (PIS) $\tilde{u}_{j}^{*}=(1,1,1)$ and the negative ideal solution (NIS) $\tilde{u}_{j}^{-}=$ $(0,0,0)$ and the distance between each alternative and the positive ideal solution and the negative solution are calculated as:

$$
\begin{aligned}
& d_{i}^{*}=\sum_{j=1}^{n} d\left(\tilde{u}_{i j}^{w}, \tilde{u}_{j}^{*}\right) \\
& d_{i}^{-}=\sum_{j=1}^{n} d\left(\tilde{u}_{i j}^{w}, \tilde{u}_{j}^{-}\right)
\end{aligned}
$$

where $i=1, \ldots, m$ and $j=1, \ldots, n$.

Then we calculate PI for each alternative as:

$$
P I_{i}=\frac{1}{2 n}\left[d_{i}^{-}+n-d_{i}^{*}\right]
$$

where $i=1, \ldots, m$, and $n$ is the number of criteria

Meanwhile for IFNs, the PI, the intuitionistic fuzzy weighted averaging (IFWA) operator proposed by [23] is used as:

$$
\begin{gathered}
\alpha_{i j}=I F W A_{\beta}\left(\alpha_{i j}^{(1)}, \alpha_{i j}^{(2)}, \ldots, \alpha_{i j}^{(t)}\right)=\beta_{1} \alpha_{i j}^{(1)} \oplus \beta_{2} \alpha_{i j}^{(2)} \oplus \ldots \oplus \beta_{t} \alpha_{i j}^{(t)} \\
=\left(1-\prod_{k=1}^{t}\left(1-\mu_{i j}^{(k)}\right)^{\beta_{k}}, \prod_{k=1}^{t}\left(v_{i j}^{(k)}\right)^{\beta_{k}}, \prod_{k=1}^{t}\left(1-\mu_{i j}^{(k)}\right)^{\beta_{k}}-\prod_{k=1}^{t}\left(v_{i j}^{(k)}\right)^{\beta_{k}}\right)
\end{gathered}
$$

where

$$
\alpha_{i j}=\left(\mu_{i j}, v_{i j}, \pi_{i j}\right), \mu_{i j}=1-\prod_{k=1}^{t}\left(1-\mu_{i j}^{(k)}\right)^{\beta_{k}}, v_{i j}=\prod_{k=1}^{t}\left(v_{i j}^{(k)}\right)^{\beta_{k}}, \pi_{i j}=\prod_{k=1}^{t}\left(1-\mu_{i j}^{(k)}\right)^{\beta_{k}}-\prod_{k=1}^{t}\left(v_{i j}^{(k)}\right)^{\beta_{k}},
$$

$i \in M, j \in N$

Then, the intuitionistic fuzzy entropy weight of each aggregated of each row of IFN matrix is defined as:

$$
\overline{\bar{w}}_{i}=-\frac{1}{n \ln 2}\left[\mu_{i} \ln \mu_{i}+v_{i} \ln v_{i}-\left(1-\pi_{i}\right) \ln \left(1-\pi_{i}\right)-\pi_{i} \ln 2\right]
$$

Here if $\mu_{i}=0, v_{i}=0, \pi_{i}=1$ then $\mu_{i} \ln \mu_{i}=0, v_{i} \ln v_{i}=0,\left(1-\pi_{i}\right) \ln \left(1-\pi_{i}\right)=0$ and if $\mu_{i}=1, v_{i}=0, \pi_{i}=$ 0 then $\mu_{i} \ln \mu_{i}=0, v_{i} \ln v_{i}=0,\left(1-\pi_{i}\right) \ln \left(1-\pi_{i}\right)=0$ respectively. Thus, the final weight/performance index(PI) for each alternative is given as:

where $\sum_{j=1}^{n} w_{i}=1$

$$
w_{i}=\frac{1-\overline{\bar{w}}_{i}}{n-\sum_{j=1}^{n} \overline{\bar{w}}_{i}}
$$




\subsection{Rank the alternatives}

Rank all the alternatives based on the performance index (PI) and the closer the PI is to 1, the better the alternative's performance.

\section{An Empirical Example}

Let us illustrate our proposed method using a hypothetical example of a car selection problem. In this calculation example, suppose that a potential buyer would like to buy a Ford car. Assuming that a single decision-maker (i.e., a buyer) is involved in this selection process, let $A_{i}(i=1,2,3)$ be cars which may be considered as: $A_{l}$ as a Fiesta, $A_{2}$ as an Escort, and $A_{3}$ as a Mondeo. A criteriawhich is the decision-maker must consider to buy a car, $\left\{C_{1}, C_{2}, C_{3}, C_{4}, C_{5}\right\}$ and is represented as price, cargo volume, maximum speed, acceleration, safety\}. Based on the above situation, the unifying procedures from Section 3 are given as follows:

\section{Step 1: Problem definitions and formulation}

According to the entire criteria, $C_{1}, C_{2}, C_{3}, C_{4}$ \{price, cargo volume, maximum speed, acceleration, $\}$ are quantitative data and $C_{5}$ \{safety $\}$ is qualitative data. Specifically, the following criteria; $C_{1}$ \{price\} and $C_{3}$ \{maximum speed $\}$ are both in interval datasets, $C_{2}$ \{cargo volume $\}$ and $C_{4}$ \{acceleration $\}$ are both in exact figures and lastly $C_{5}$ \{safety is in linguistic form. Table 3 shows all the alternatives and criteria fora car selection problem.

Table 3. The Alternatives and Criteria for aCar Selection Problem

\begin{tabular}{cccccc}
\hline & $\begin{array}{c}\text { Price, } C_{1} \\
(\mathrm{RM} \text { '000) }\end{array}$ & $\begin{array}{c}\text { Cargo volume, } C_{2} \\
\left(\mathrm{dm}^{3}\right)\end{array}$ & $\begin{array}{c}\text { Maximum speed, } C_{3} \\
(\mathrm{~km} / \mathrm{h})\end{array}$ & $\begin{array}{c}\text { Acceleration, } C_{4} \\
(\mathrm{Sec})\end{array}$ & $\begin{array}{c}\text { Safety, } C_{5} \\
(\text { linguistic })\end{array}$ \\
\hline Fiesta, $A_{1}$ & {$[85,90]$} & 250 & {$[153,180]$} & 15.3 & Medium $(M)$ \\
\hline Escort, $A_{2}$ & {$[110,145]$} & 380 & {$[177,195]$} & 12.3 & High $(H)$ \\
\hline Mondeo, $A_{3}$ & {$[135,180]$} & 480 & {$[195,210]$} & 11.1 & Very High $(V H)$ \\
\hline Note USD1 $=$ RM4 (Approx $)$
\end{tabular}

\subsection{Unifying Process using TFNs}

\section{Step 2: Unifying process}

We have identified $C_{1}$ \{price $\}$ and $\mathrm{C} 4$ \{acceleration $\}$ as cost criteria,while $C_{2}$ \{cargo volume $\}$ and $C_{3}$ \{maximum speed $\}$ are benefit criteria.Since $C_{2}$ \{cargo volume and $C_{4}$ \{acceleration\} are both represented by crisp values, then the membership function was constructed to derive the membership values. Furthermore $C_{1}$ \{price\} and $C_{3}$ \{maximum speed \} are represented by intervals, so we converted them into TFNs after consulting an expert. For example, $C_{1}$ \{price\} of A1 $(85,87,90)$ represents (standard, premium, luxury) prices. Meanwhile, $C_{3}$ \{maximum speed $\}$ is represented by TFNs as in definition 1 (minimum possible value, most possible value, maximum possible value). For qualitative input datasets, $C_{5}$ \{safety\}, we constructed the membership functions based on linguistic values for criteria rating purposes (see Table 1). Then two methods were employed to derive the score values.

i. The original data is represented by crisp values,so as per feedback from the expert; the membership function was constructed to derive the membership values.

ii. The original data is represented by interval and linguistic values, so the advantages of triangular fuzzy numbers (TFNs) were employed and the unifying processes were derived based on either the cost criteria from (3) or benefitcriteria from (4).

All the unifying processes from raw dataset into TFNs in the range of $[0,1]$ are shown in Table 4.

Table 4. The Unifying Process from Raw Dataset into TFNs

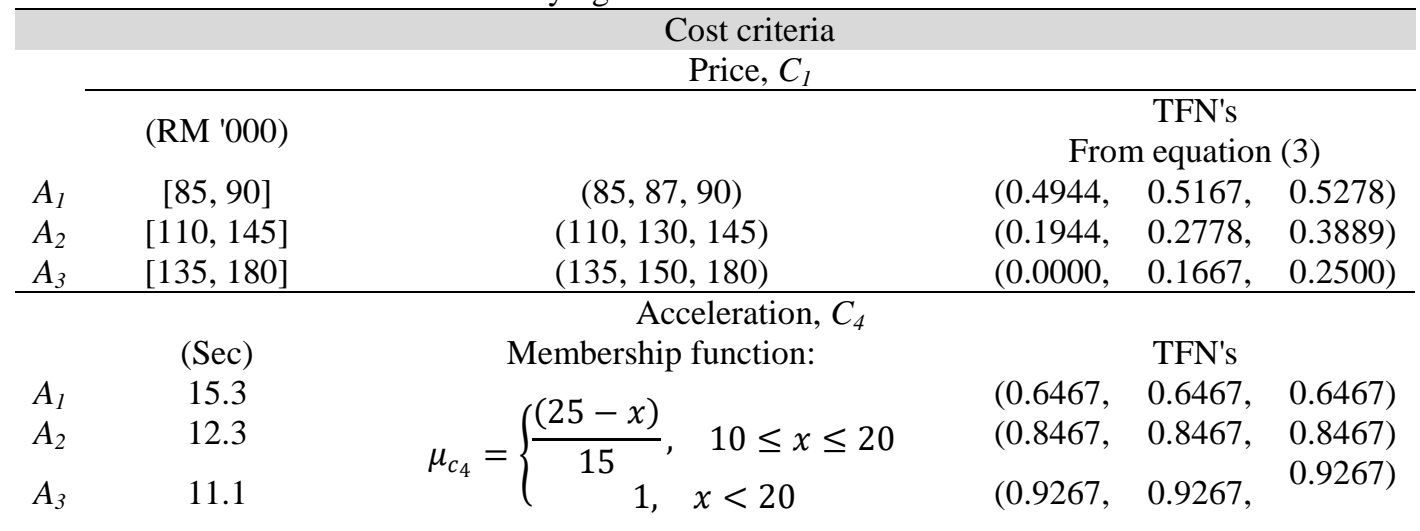




\begin{tabular}{|c|c|c|c|c|c|c|c|}
\hline \multicolumn{8}{|c|}{ Benefit criteria } \\
\hline & $\left(\mathrm{dm}^{3}\right)$ & \multicolumn{3}{|c|}{$\begin{array}{l}\text { Cargo volume, } C_{2} \\
\text { Membership function: }\end{array}$} & \multicolumn{3}{|c|}{ TFN's } \\
\hline$A_{1}$ & 250 & \multicolumn{2}{|c|}{$((x-240)$} & \multirow[b]{2}{*}{$200 \leq x \leq 500$} & \multirow{3}{*}{$\begin{array}{l}(0.0408, \\
(0.5714, \\
(0.9796\end{array}$} & \multirow{3}{*}{$\begin{array}{l}0.0408, \\
0.5714, \\
0.9796,\end{array}$} & $0.0408)$ \\
\hline$A_{2}$ & 380 & $\mu_{c_{2}}=$ & 245 & & & & $0.5714)$ \\
\hline$A_{3}$ & 480 & & & $500<x$ & & & $0.9796)$ \\
\hline
\end{tabular}

$(\mathrm{km})$

\begin{tabular}{lll}
$A_{1}$ & {$[153,180]$} & $(153,170,180)$ \\
$A_{2}$ & {$[177,195]$} & $(177,185,195)$ \\
$A_{3}$ & {$[195,210]$} & $(195,200,210)$ \\
\hline
\end{tabular}

TFN's

From equation (2)

$(0.9000, \quad 0.8095, \quad 0.8571)$

$(0.8429, \quad 0.8810, \quad 0.9286)$

$(0.9286, \quad 0.9524, \quad 1.0000)$

\begin{tabular}{cccccc}
$A_{3}$ & {$[195,210]$} & $(195,200,210)$ & $(0.9286$, & 0.9524, & $1.0000)$ \\
\hline & linguistic & Safety, $C_{5}$ & & \\
\hline$A_{1}$ & Medium $(M)$ & From Table 1 & TFN's \\
$A_{2}$ & High $(H)$ & & $(0.3000$, & 0.5000, & $0.7000)$ \\
$A_{3}$ & Very High $(V H)$ & & $(0.7000$, & 0.8000, & $0.9000)$ \\
Note: & USD1 $=$ RM4(Approx.) & & $(0.8000$, & 0.9000, & $1.000)$ \\
\hline
\end{tabular}

The higher the value of $\tilde{u}_{i j}$ for $\omega_{1}$, the better it is for DMs and the lower the value of $\tilde{u}_{i j}$ for $\omega_{2}$, the better it is for the DMs.

\section{Step 3: Performance matrix}

Since $C_{2}$ \{cargo volume $\}$ and $C_{4}\{$ acceleration $\}$ are exact values, hence we derivedtheir corresponding values to TFNs. The overall scores were expressed in the performance matrix as shown in Table 5.

Table 5. Performance Matrix by TFNs

\begin{tabular}{cccccc}
\hline & Price, $C_{1}$ & Cargo volume, $C_{2}$ & Max. speed, $C_{3}$ & Acceleration, $C_{4}$ & Safety, $C_{5}$ \\
\hline \multirow{2}{*}{$A_{1}$} & $(0.4944,0.5167$, & $(0.0408,0.0408$, & $(0.9000,0.8095$, & $(0.6467,0.6467$, & $(0.3000,0.5000$, \\
& $0.5278)$ & $0.0408)$ & $0.8571)$ & $0.6467)$ & $0.7000)$ \\
$A_{2}$ & $(0.1944,0.2778$, & $(0.5714,0.5714$, & $(0.8429,0.8810$, & $(0.8467,0.8467$, & $(0.7000,0.8000$, \\
& $0.3889)$ & $0.5714)$ & $0.9286)$ & $0.8467)$ & $0.9000)$ \\
$A_{3}$ & $(0.000,0.1667$, & $(0.9796,0.9796$, & $(0.9286,0.9524$, & $(0.9267,0.9267$, & $(0.8000,0.9000$, \\
& $0.2500)$ & $0.9796)$ & $1.0000)$ & $0.9267)$ & $1.000)$ \\
\hline
\end{tabular}

\section{Step 4: Performance index}

The distance to the PIS and NIS were calculated to obtain the performance index of each alternative using (16) (17), respectively. Table 6 shows the performance index of the alternatives

Table 6. The Distance to the PIS and NIS Performance Matrix and PI of Alternatives

\begin{tabular}{lccc}
\hline & Distance to the positive ideal solution & Distance to the negative ideal solution & PI \\
\hline$A_{1}$ & 2.3612 & 2.5830 & 0.5222 \\
\hline$A_{2}$ & 1.6364 & 3.4050 & 0.6769 \\
\hline$A_{3}$ & 1.1397 & 3.9443 & 0.7805 \\
\hline
\end{tabular}

\section{Step 5: Performance and ranking of the alternatives}

Table 7. Performance Index (PI) and Ranking of Alternatives by TFNs

\begin{tabular}{cccccc}
\hline \multicolumn{2}{c}{ Fiesta, $A_{1}$} & \multicolumn{2}{c}{ Escort, $A_{2}$} & \multicolumn{2}{c}{ Mondeo, $A_{3}$} \\
\hline PI & Order & PI & Order & PI & Order \\
\hline 0.5222 & 3 & 0.6769 & 2 & 0.7805 & 1 \\
\hline
\end{tabular}

Thus, we ranked all the alternatives using (18) and the results areas shown in Table 7. Apparently, $\mathrm{A}_{3}\{$ Mondeo $\}$ is the best option due to the highest PI as compared to other alternatives. The rank is $A_{3}>A_{2}>A_{1}$ where '>' means 'more superior' or 'preferred'.

\subsection{Unifying Process using IFNs}

Based on Step 1 from Section 4 above, now we proceed directly to the unifying process using IFNs as: 


\section{Step 2: Unifying process}

At this stage, two methods were employed to derive the score values:

i. The original data is represented by crisp values: $C_{4}$ \{acceleration $\}$ and $C_{2}$ \{cargo volume $\}$ and also interval values: $C_{1}$ \{price $\}$ and $C_{3}$ \{maximum speed , so as per feedback from the expert; the membership function was constructed to derive the IFNs and normalize its cost-benefit criterion using (7)-(12).

ii. The original data is represented bylinguistic variables, $C_{5}$ \{safety $\}$ (see Table 2 ) for its IFNs and we normalized its cost-benefit criterion using (13) - (14).

The unifying process from raw datasets into IFNs is shown in Table 8 below.

Table 8. The Unifying Process from Raw Dataset into IFNs

\begin{tabular}{|c|c|c|c|c|}
\hline \\
\hline & \multicolumn{4}{|c|}{ Cost criteria } \\
\hline & \multicolumn{4}{|c|}{ Price, $C_{l}$} \\
\hline & (RM '000) & & IFN's & \\
\hline$A_{1}$ & {$[85,90]$} & $(0.6689$ & 0.1720 & $0.1591)$ \\
\hline$A_{2}$ & {$[110,145]$} & $(0.4152$ & 0.3602 & $0.2246)$ \\
\hline$A_{3}$ & {$[135,180]$} & $(0.3345$ & 0.4787 & $0.1869)$ \\
\hline \multicolumn{5}{|c|}{ Acceleration, $C_{4}$} \\
\hline & $(\mathrm{Sec})$ & & IFN's & \\
\hline$A_{l}$ & 15.3 & $(0.4742$ & 0.5258 & $0.0000)$ \\
\hline$A_{2}$ & 12.3 & $(0.5899$ & 0.4101 & $0.0000)$ \\
\hline$A_{3}$ & 11.1 & $(0.6536$ & 0.3464 & $0.0000)$ \\
\hline \multicolumn{5}{|c|}{ Benefit criteria } \\
\hline \multicolumn{5}{|c|}{ Cargo volume, $C_{2}$} \\
\hline & $\left(\mathrm{dm}^{3}\right)$ & & IFN's & \\
\hline$A_{1}$ & 250 & $(0.3781$ & 0.6219 & $0.0000)$ \\
\hline$A_{2}$ & 380 & $(0.5746$ & 0.4254 & $0.0000)$ \\
\hline$A_{3}$ & 480 & $(0.7259$ & 0.2741 & $0.0000)$ \\
\hline \multicolumn{5}{|c|}{ Maximum speed, $C_{3}$} \\
\hline & $(\mathrm{km})$ & & IFN's & \\
\hline$A_{l}$ & {$[153,180]$} & $(0.4521$ & 0.4090 & $0.1389)$ \\
\hline$A_{2}$ & {$[177,195]$} & $(0.5230$ & 0.3598 & $0.1172)$ \\
\hline$A_{3}$ & {$[195,210]$} & $(0.5762$ & 0.3105 & $0.1133)$ \\
\hline \multicolumn{5}{|c|}{ Safety, $C_{5}$} \\
\hline & linguistic & & IFN's & \\
\hline$A_{l}$ & Medium $(M)$ & $(0.5000$ & 0.3500 & $0.1500)$ \\
\hline$A_{2}$ & $\operatorname{High}(H)$ & $(0.6500$ & 0.2500 & $0.1000)$ \\
\hline$A_{3}$ & Very High $(V H)$ & $(0.7500$ & 0.1500 & $0.1000)$ \\
\hline
\end{tabular}

\section{Step 3: Performance matrix}

The overall scores were expressedin the performance matrix as shown in Table 9.

Table 9. Performance Matrix by IFNs

\begin{tabular}{cccccc}
\hline & Price, $C_{l}$ & Cargo volume, $C_{2}$ & Max. speed, $C_{3}$ & Acceleration, $C_{4}$ & Safety, $C_{5}$ \\
\hline \multirow{2}{*}{$A_{I}$} & $(0.6689,0.1720$, & $(0.3781,0.6219$, & $(0.4521,0.4090$, & $(0.4742,0.5258$, & $(0.5000,0.3500$, \\
& $0.1591)$ & $0.0000)$ & $0.1389)$ & $0.0000)$ & $0.1500)$ \\
$A_{2}$ & $(0.4152,0.3602$, & $(0.5746,0.4254$, & $(0.5230,0.3598$, & $(0.5899,0.4101$, & $(0.6500,0.2500$, \\
& $0.2246)$ & $0.0000)$ & $0.1172)$ & $0.0000)$ & $0.1000)$ \\
$A_{3}$ & $(0.3345,0.4787$, & $(0.7259,0.2741$, & $(0.5762,0.3105$, & $(0.6536,0.3464$, & $(0.7500,0.1500$, \\
& $0.1869)$ & $0.0000)$ & $0.1133)$ & $0.0000)$ & $0.1000)$ \\
\hline
\end{tabular}

\section{Step 4: Performance index}

We calculated the aggregated matrix and entropy weight to obtain PI of each alternative using (19) - (21). Table 10 shows the performance index of the alternatives. 
Table 10. The Aggregated Matrix, Entropy Weight and PI of Alternatives

\begin{tabular}{|c|c|c|c|c|c|}
\hline & \multicolumn{3}{|c|}{ Aggregated matrix } & Entropy weight & Final weight/Performance index (PI) \\
\hline Fiesta, $A_{l}$ & $(0.9703$, & 0.0081 , & $0.0216)$ & 0.02964596 & 0.3299394 \\
\hline Escort, $A_{2}$ & (0.9830, & 0.0057 , & $0.0114)$ & 0.020539634 & 0.33303573 \\
\hline Mondeo, $A_{3}$ & (0.9933, & 0.0021 , & $0.0046)$ & 0.00880753 & 0.33702487 \\
\hline
\end{tabular}

\section{Step 5: Performance and ranking of the alternatives}

Table 11.Performance Index (PI) and Ranking of Alternatives by IFNs

\begin{tabular}{cccccc}
\hline \multicolumn{2}{c}{ Fiesta, $A_{1}$} & \multicolumn{2}{c}{ Escort, $A_{2}$} & \multicolumn{2}{c}{ Mondeo, $A_{3}$} \\
\hline PI & Order & PI & Order & PI & Order \\
\hline 0.3299 & 3 & 0.3330 & 2 & 0.3370 & 1 \\
\hline
\end{tabular}

We ranked all thealternatives based on their PI. From Table 11, the results show that the final ranking remained unchanged and is similar to the result when we used the unifying by TFNs method (subsection 4.1): $A_{3}>A_{2}>A_{1}$ where '>' means 'more superior' or 'preferred'.

\section{Conclusion}

In this paper, we have presented the unifying process using TFNs and IFNs which are highly beneficial in terms of applicability and efficiency for uniform data from diverse input datasets from the fuzzy and intuitionistic fuzzy perspectives. It is very useful in a variety of real-world decision-making situations which involve diverse input datasets. In particular, the TFNs based on the cost-benefit criterion is an approach that can adequately manage the imprecision and uncertainty of the human decision judgment and provide flexibility to DMs. Meanwhile, the IFNs based on the cost-benefit criterionapproach is used to normalize non-homogenous criteria The approach proposed in this studyhasthe ability to overcome the drawbacks of individual TFN and IFN approaches to deal with diversedatasets such as crisp, interval and linguistic terms. Moreover, the proposed approach in this study also offersa versatile judgment, has clear procedures and also has great potential as a unifying tool for decision-making purposes. Thus, it helps the decision-makers (DMs) to make their decisionseasily and in a systematic manner. For future work, the sensitivity analysis could be equipped in the proposed approach to ensure the results in any case studies are more feasible and acceptable in terms of their final outcomes.

\section{Acknowledgements}

The authors acknowledge the financial support from FRGS grant Ref. No.: 600-RMI/FRGS 5/3(0084/2016) from the Ministry of Higher Education (MoHE) Malaysia and UniversitiTeknologi MARA (UiTM), Malaysia.

\section{References}

[1] Suwelack, K., \& Dominik W. An approach to unify the appraisal framework for biomass conversion systems. Biomass and Bioenergy, 83, 2015, 354-365.

[2] S-Vela, M. V., Moreo, R. T., Sa'enz, F. T., \&Zamora-Izquierdo, M. A. An application of a fuzzy classifier extracted from data for collision avoidance support in road vehicles. Engineering Applications of Artificial Intelligence, 26, 2013, $173-183$.

[3] Bricq, S. , Collet, C., \&Armspach, J. P. Unifying framework for multimodal brain MRI segmentation based on Hidden Markov Chains. Medical Image Analysis, 12, 2008, 639-652.

[4] Kaneiwa, K. A rough set approach to multiple dataset analysis. Applied Soft Computing, 11, 2011, $2538-2547$.

[5] Jun, L., Li, L. X., \&Jun, W. A problem Classification Approach in Business Service Management. International Conference on Computer Application and System Modeling, IEEE, 10, 2010, 354-356.

[6] Zamali, T. Fuzzy hybrid multi-criteria decision-making model for municipal solid waste disposal selection. Universiti Malaysia Terengganu, Malaysia, PhD Thesis (unpublished). 2009.

[7] Chen, S. J., \& Hwang, C. L. (1992). Fuzzy Multiple Attribute Decision Making Methods and Applications. Springer-Verlag, Berlin, Heidelberg.

[8] Kannan, D., Khodaverdi, R., Olfat, L., Jafarian, A., \&Diabat, A. Integrated fuzzy multi criteria decision making method and multiobjective programming approach for supplier selection and order allocation in a green supply chain. Journal of Cleaner Production, 47, 2013, 355-367.

[9] Damghani, K. K., \&Nezhad S. S. (2013). A hybrid fuzzy multiple criteria group decision making approach for sustainable project selection. Applied Soft Computing, 13, 339-352.

[10] Fenton, N., \&Neil, M. Making decisions: using Bayesian nets and MCDA. Knowledge Based Systems, 14, $2001,307-325$.

[11] Yager, R. R. Fuzzy modeling for intelligent decision making under uncertainty. IEEE Transactions on Systems Man and Cybernetics - Part B: Cybernetics, 30, 2000, 60-70.

[12] Yager, R. R.. On the valuation of alternatives for decision-Making under uncertainty. International Journal of Intelligent Systems, 17, 2002, 687-707.

[13] Xiaodan, W., Jingwei, Z., Yafei, S., \&Lei L. Combination of unreliable evidence sources in intuitionistic fuzzy MCDM framework. Knowledge-Based Systems, 97, 2016, 24-39.

[14] Miguel, M-S., Maria, R., \&Miguel, D. Unifying fuzzy controller for IEQ: implementation in a Raspberry Pi. Atlantis Press, 1034 1039 (2015) 
[15] Michael, S., \&Ralf, S. Arguments and Misunderstandings: Fuzzy Unification for Negotiating Agents. Electrical Notes in Theoretical Computer Science, 70(5), 2002.

[16] Zadeh, L. A. Fuzzy Sets eds. R R Yager, S Ovchinnikov, R M Tong, and H T Nguyen. Information and Control 8(3), $1965,338-53$.

[17] Fenton, N., \&Wang, W. Risk and confidence analysis for fuzzy multicriteria decision making. Knowledge Based-Systems, 19, 2006, 430-437.

[18] Atanassov, K. T.Intuitionistic fuzzy sets, Original Research Article.Fuzzy Sets and Systems, 20(1), 1986, 87-96.

[19] Guo, J. Hybrid Multiattribute Group Decision Making Based on Intuitionistic Fuzzy Information and GRA Method.Hindawi Publishing Corporation ISRN Applied Mathematics, Article ID 146026, 10 pages (2013).

[20] Zeshui, X. \& Hui, H. Projection Models for Intuitionistic Fuzzy Multiple Attribute Decision Making. International Journal of Information Technology \& Decision Making 9(2), 2010, 267-80.

[21] Chen, C. T. Extensions of the TOPSIS for group decision-making under fuzzy environment. Fuzzy Sets and Systems, 114, 1-9, 2000.

[22] Hwang, C. L., \&Yoon, K. Multiple Attributes Decision Making Methods and Application. Springer-Verlag, Berlin, Heidelberg. 1981.

[23] Zeshui, X. Some Similarity Measures of Intuitionistic Fuzzy Sets and Their Applications to Multiple Attribute Decision Making. Fuzzy Optimization and Decision Making 6(2), 2007, 109-21. 